\title{
MUON TRIDENTS AT NAL
}

M. J. Tannenbaum

Harvard University

July 12, 1968

The direct production of muon pairs by muons in the field of a heavy nucleus is interesting because it probes the muon-muon interaction. The only other way to probe this interaction is with muon $g-2$ experiments. At the present time, the experimental value of $g-2$ for the muon is higher than the theory by $800 \pm 300 \mathrm{ppm}$ while the value for the electron deviates from theory by less than $60 \mathrm{ppm} .{ }^{1}$ Taken at face value, these results could indicate an anomalous muon-muon interaction with amplitude $0.4 \times 10^{-3}$ of the electromagnetic muon-muon amplitude at $\mathrm{q}^{2}=\mathrm{m \mu} \mu^{2}$. If this anomalous interaction were represented by a heavy photon of coupling constant $f$ and mass $M$ then the $g-2$ result gives $\mathrm{f}^{2} / \mathrm{M}^{2}=0.0008 \pm 0.0003 \mathrm{GeV}^{-2}$; or $1 / \mathrm{M}^{2}=0.11 \pm 0.04$ $\mathrm{GeV}^{-2}$ if $\mathrm{f}^{2}=\alpha$.

If you try to take this model literally, it implies that for coupling strength $\alpha$ or weaker there should exist heavy neutral particles of mass $3 \mathrm{GeV}$ or less!

These "heavy photons" could affect muon tridents in two ways. First, in the spacelike diagrams "heavy photon" exchange will interfere

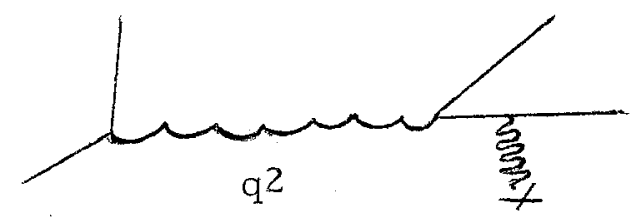


with the usual photon exchange and will modify the $\mathrm{q}^{2}$ distribution from $1 / q^{4}$ to

$$
\frac{1}{q^{4}}\left(2-\frac{1}{\left(1+q^{2} / M^{2}\right)}\right)^{2} \text {. }
$$

This is the effect presumably observed in the $g-2$ experiment. In the trident reaction, however, there are also timelike contributions; real heavy photons could be produced by a Bremsstrahlung-like process. The heavy photon would then decay into a muon pair, giving the event the appearance of a muon trident. Even though a heavy nucleus is used as a target, it will act incoherently when the mass of the produced particle is comparable to the incident energy because of the large recoil momentum required

$$
t=\left(\frac{M_{x}^{2}}{2 E_{x}}\right)^{2}
$$

The big advantage of a $100-\mathrm{BeV}$ muon beam will be that $3-\mathrm{BeV}$ particles can be produced with $t<(0.05 \mathrm{GeV})^{2}$, so that they could be produced coherently from a heavy nucleus like lead.

In order to find out which experiment is best to detect such a hypothetical heavy photon, we must know to which other particles it couples. If it couples to electrons as well as muons, or to electrons only, then the best experiments to do are ee scattering or e $\bar{e} \rightarrow \mu \bar{\mu}$ in colliding beams. Experiments to be conducted at the CEA in 1970 or 1971 should obtain a sensitivity. 


$$
\Delta\left(\frac{1}{M^{2}}\right)= \pm 0.002 \mathrm{GeV}^{-2}
$$

which is 200 times more sensitive than the $\mathrm{g}-2$ result,

$$
\frac{1}{\mathrm{M}^{2}}=775.0 \pm 0.04 \mathrm{GeV}^{-2}
$$

If the force couples to muons only, then muon trident experiments will serve as a complement to the $g-2$ experiments, first as a confirmation, and secondly because the hypothetical particles could be produced and observed experimentally. One interesting possibility is that a muon-muon force couples to "mu-ness" and not just to electric charge (in an analogy to the strong force which couples to $\pi^{\circ}$ as well as $\pi^{ \pm}$). This would mean that mu-neutrino pairs couple to muon pairs so that an incident mu-neutrino could produce a pair of muons in the field of a heavy nucleus i. e., a neutrino trident. This reaction is the cleanest way to search for a force coupled to "mu-ness" because the only source of background is the weak interaction. This will be discussed in detail elsewhere.

Muon trident experiments would be most useful to observe a muon-muon force which is coupled only to charged muons. As previously mentioned, this force would modify the $\mathrm{q}^{2}$ distribution of the trident, and, if the incident muon energy were much greater than the mass of the hypothetical particle, then the particle could be produced and its muon pair decay would drastically alter the invariant mass 
distribution of the trident. In either case, an increase in energy from the $10-20 \mathrm{GeV}$ muons now available, to the $100-200 \mathrm{GeV}$ muons at NAL would be an improvement.

For the actual production of particles, increasing the energy would allow higher masses to be produced, but more importantly the recoil momentum necessary to produce a particle of a given mass would be reduced. Another gain is that the total muon trident cross section increases roughly linearly with energy in the region 20-100 $\mathrm{BeV}$, so that you can get more tridents per incident muon at $100 \mathrm{BeV}$. For instance, at $17 \mathrm{BeV}$ the total muon trident cross section on carbon is $0.012 \mu \mathrm{b}$ while at $100 \mathrm{BeV}$ it is $0.045 \mu \mathrm{b}$. The $\mathrm{q}^{2}$ distribution of tridents drops like $1 / \mathrm{q}^{4}$ and even at modest incident energies, you run out of cross section at values of $q^{2}$ much less than the maximum obtainable. Thus, increasing the incident energy helps you only because the cross section rises, so that you get more events at a given $\mathrm{q}^{2}$. For a $17-\mathrm{GeV}$ experiment now under consideration at Brookhaven with a flux of $10^{6} \mu / \mathrm{sec}$ and a 20 -inch thick lead target, a precision

$$
\Delta\left(\frac{1}{\mathrm{M}^{2}}\right)= \pm 0.03 \mathrm{GeV}^{-2} \text {, }
$$

is obtainable in a 400 -hour run. If we scale up the cross section and use $10^{7} \mu / \mathrm{sec}$ as a reasonable flux to expect at NAL, then a 400 -hour run with $100-\mathrm{BeV}$ incident muons on the same target would give a precision 


$$
\Delta\left(\frac{1}{\mathrm{M}^{2}}\right)= \pm 0.005 \mathrm{GeV}^{-2}
$$

One thing to stress for muon beams at NAL is the importance of making very well collimated and very well momentum-defined muon beams even at the expense of intensity. The intensities quoted by Yamanouchi ${ }^{2}\left(10^{8} / \mathrm{sec}\right)$ are already a factor of 10 to 100 higher than can be used by present day techniques. At $100 \mathrm{GeV}$, all scattering angles will be small so that any experiment which measures the angle of a scattered particle will need a detector essentially in the beam. Unless the beam is very well defined spatially so that is can be passed through a hole in the detector, the stray beam tracks will completely obscure the event of interest.

\section{REFERENCES}

${ }^{1}$ H. R. Crane.

${ }^{2}$ T. Yamanouchi, NAL Summer Study Report B. 2-68-38, 1968. 